\title{
Hábitat y distribución de Aphelocheirus murcius Nieser \& Millán, 1989 (Hemiptera: Aphelocheiridae) en el norte de la Península Ibérica
}

\author{
David Miguélez y Luis Felipe Valladares*
}

Departamento de Biodiversidad y Gestión Ambiental (Zoología), Facultad de Ciencias Biológicas y Ambientales, Universidad de León, 24071 León (España).

* Autor responsable de la correspondencia: lfvald@unileon.es

Recibido: 4/3/10

Aceptado: 29/6/10

\begin{abstract}
Habitat and distribution of Aphelocheirus murcius Nieser \& Millán, 1989 (Hemiptera: Aphelocheiridae) in the North of the Iberian Peninsula

From samples collected mostly from rivers in the Upper Ebro river basin, the distribution area is expanded for Aphelocheirus murcius, an endemic water bug of the Iberian Peninsula with a very narrow and little known distribution range. Moreover, new data are provided on habitat preferences, water quality, physical and chemical variables, submerged vegetation, and preservation level of the river banks. These characteristics allow the assumption that Aphelocheirus murcius is a river species of well preserved, middle reaches.
\end{abstract}

Key words: Water bugs, Aphelocheirus murcius, Spain, Ebro river basin, distribution, habitat.

\section{RESUMEN}

Hábitat y distribución de Aphelocheirus murcius Nieser \& Millán, 1989 (Hemiptera: Aphelocheiridae) en el norte de la Península Ibérica

A partir de muestreos realizados mayoritariamente en ríos de la cuenca alta del Ebro se amplía la distribución de Aphelocheirus murcius, un hemíptero acuático endémico de la Península Ibérica y con un área de distribución muy reducida y poco conocida. Además, se aportan nuevos datos sobre sus preferencias de hábitat, calidad del agua, parámetros fisicoquímicos, vegetación sumergida y grado de conservación de las riberas. Estas características permiten concluir que se trata de una especie fluvial de tramos medios y bien conservados.

Palabras clave: Chinches acuáticas, Aphelocheirus murcius, España, cuenca río Ebro, distribución, hábitat.

\section{INTRODUCCIÓN}

Los hemípteros acuáticos de la familia Aphelocheiridae presentan una amplia distribución y una elevada diversidad en la región Paleártica, contando con unas 60 especies (Aukema \& Rieger, 1995). Su representación en la mayor parte de Europa occidental se limita únicamente a Aphelocheirus aestivalis (Fabricius, 1794), especie a la que se habían atribuido todas las citas ibéricas de
Aphelocheirus anteriores a 1989 (Seabra, 1926, 1939; Fernández, 1982; Murillo, 1985; Niesser \& Montes, 1984; Baena \& Vázquez, 1986). Sin embargo, la presencia ibérica de A. aestivalis necesita confirmación o incluso debería ser descartada (Nieser et al., 1994; Carbonell, 2009) ya que probablemente estos registros sean atribuibles a cualquiera de las dos especies endémicas de la Península Ibérica descritas ese año: Aphelocheirus occidentalis Nieser \& Millán, 1989 y Aphelo- 
cheirus murcius Nieser \& Millán, 1989. Las citas confirmadas de A. murcius se concentran en el suroeste de la provincia de Albacete, en los ríos Tús y Mundo, donde se describió la especie (Nieser \& Millán, 1989; Carbonell, 2009). Fuera de esta provincia sólo se ha citado en las provincias de Álava y Guipúzcoa (Millán et al., 2002).

En el presente trabajo se aportan nuevos datos sobre el área de distribución de A. murcius en el norte de España y se profundiza en el conocimiento de su hábitat.

\section{MATERIAL Y MÉTODOS}

Las capturas proceden de estudios relacionados con el uso de macroinvertebrados como indicadores de la calidad del agua, realizados en varios ríos de las cuencas hidrográficas del Ebro y del Norte durante los años 2006 y 2008 en la provincia de Burgos. El número de estaciones de muestreo estudiadas es de 99, repartidas en las subcuencas de los ríos Ebro (22), Rudrón (9), Trifón (2), Nela (12), Trueba (15), Jerea (8), Ranera (2), Purón (2), Oroncillo (5), Ayuda (8) y Cadagua (14), todas ellas pertenecen a la cuenca del río
Ebro, excepto la del río Cadagua, afluente del río Nervión. Las estaciones con capturas se reflejan en orden descendente en la Tabla 1.

Los datos físico-químicos del agua se tomaron una vez durante el mes de julio, cuando el estiaje es acusado y permite detectar con mayor precisión posibles perturbaciones. Para este análisis se siguieron los protocolos estandarizados en este tipo de estudios (Jáimez-Cuéllar, et al., 2002). Mediante sonda multiparamétrica (Hach HQ40d) se obtuvieron medidas directas en el campo de los parámetros temperatura, oxígeno disuelto, porcentaje de saturación de oxígeno en agua, conductividad y pH. Por medio de espectofotometría y métodos colorimétricos (espectrofotómetro Hach DR/2000) se han analizado los parámetros turbidez, dureza, sulfatos, nitratos, nitritos, ión amonio y fosfatos.

Los macroinvertebrados fueron recogidos mediante una manga rectangular de entomología acuática de $250 \mu \mathrm{m}$ de luz de malla. Cada tramo evaluado tenía una longitud aproximada de $100 \mathrm{~m}$ donde se muestrearon todos los hábitats presentes durante el tiempo necesario hasta que aparentemente no aparecían nuevos taxones (Alba-Tercedor et al., 2002; Jáimez-Cuéllar

Tabla 1. Material estudiado (n: ninfas). Studied material ( $n$ : nymphs).

\begin{tabular}{|c|c|c|c|c|}
\hline Localidad & Cauce & U.T.M. & Fecha & Capturas \\
\hline Bárcenas & Trueba & 30T 4537814772184 & $21 / 03 / 2006$ & $1(1 \mathrm{n})$ \\
\hline Orbaneja del Castillo & Ebro & 30T 4354454743170 & 07/07/2008 & 38 (5 ○ $\sigma^{\top}, 4$ 우, 29 n) \\
\hline Orbaneja del Castillo & Ebro & 30T 4356914742600 & 07/07/2008 & $51\left(4 \odot^{2} \sigma^{2}, 7\right.$ 우, 40 n) \\
\hline Escalada & Ebro & 30T 4365124740278 & 07/07/2008 & $71\left(4 \sigma^{x} \sigma^{x}, 4\right.$ 우, 63 n) \\
\hline Valdelateja & Ebro & 30T 4383154737453 & 07/07/2008 & 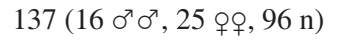 \\
\hline Pesquera de Ebro & Ebro & $30 \mathrm{~T} 4415864738860$ & 07/07/2008 & $17(1$ ㅇ, $16 \mathrm{n})$ \\
\hline Pesquera de Ebro & Ebro & 30T 4412094740166 & 07/07/2008 & 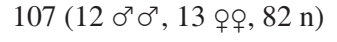 \\
\hline Colina & Ebro & $30 \mathrm{~T} 4406364741616$ & 08/07/2008 & $45\left(4 \sigma^{x} \sigma^{x}, 5\right.$ 웅, 36 n) \\
\hline Villanueva de Rampalay & Ebro & 30T 4403964743552 & 08/07/2008 & $403\left(10 \sigma^{7} \sigma^{7}, 17\right.$ 우우, 376 n) \\
\hline Tubilleja & Ebro & 30T 4420434745417 & 08/07/2008 & $91\left(2 \sigma^{7} \sigma^{7}, 6\right.$ 웅, 83 n) \\
\hline Cidad de Ebro & Ebro & 30T 4417144747675 & 09/07/2008 & $144\left(6 \sigma^{x} \sigma^{x}, 6\right.$ 우, 132 n) \\
\hline Cidad de Ebro & Ebro & 30T 4412174748751 & 09/07/2008 & 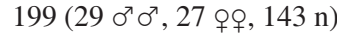 \\
\hline Manzanedillo & Ebro & 30T 4434134749431 & 09/07/2008 & $176\left(13 \sigma^{\pi} \sigma^{7}, 15\right.$ 우우, $\left.148 \mathrm{n}\right)$ \\
\hline Rioseco & Ebro & 30T 4456274749026 & 09/07/2008 & $119\left(5 \sigma^{x} \sigma^{x}, 10\right.$ 우, 104 n) \\
\hline Remolino & Ebro & 30T 4503694748735 & $10 / 07 / 2008$ & $50\left(1 \sigma^{7}, 4\right.$ 우, $\left.45 \mathrm{n}\right)$ \\
\hline Valdenoceda & Ebro & 30T 4513554744380 & $10 / 07 / 2008$ & $2(2 n)$ \\
\hline Población de Valdivielso & Ebro & 30T 4560314740826 & $10 / 07 / 2008$ & $8(1$ o, $7 \mathrm{n})$ \\
\hline Frías & Ebro & 30T 4765254735097 & $10 / 07 / 2008$ & $1(1 \mathrm{n})$ \\
\hline
\end{tabular}


et al., 2006); estos mangueos se realizaban durante 5 o 6 minutos. Los ejemplares se conservaron en etanol al $70 \%$ para su identificación en el laboratorio. El nivel taxonómico al que fueron determinados los macroinvertebrados fue el de familia, excepto Oligochaeta e Hydracarina. Se calcularon los índices IBMWP (Alba-Tercedor $e t$ $a l .$, 2002), IASPT (valor medio por taxón), riqueza de taxones (los empleados para el IBMWP) y riqueza EPT (número total de familias de los órdenes Ephemeroptera, Plecoptera y Trichoptera). Para el cálculo de estos índices, del índice de calidad del bosque de ribera (QBR) y de algunos parámetros del índice de hábitat fluvial (IHF) se siguieron los protocolos recogidos en Jáimez-Cuéllar et al. (2002).

\section{RESULTADOS Y DISCUSIÓN}

Hasta la actualidad se conocían un total de doce citas peninsulares de A. murcius, que se localizan en ocho cuadrículas UTM de $10 \times 10 \mathrm{~km}$.
Con los muestreos del alto Ebro se amplía el conocimiento a 18 nuevas localidades (Tabla 1), todas ellas en la provincia de Burgos. Estos registros ocupan seis nuevas cuadrículas que amplían el área de distribución hacia el oeste, dentro del núcleo norteño de distribución de esta especie en la Península Ibérica (Fig. 1). Todas las localidades, exceptuando una en la cabecera del río Trueba, se encuentran en el río Ebro, lo que supone la presencia de esta especie en un tramo de casi $100 \mathrm{~km}$. Destaca, por tanto, su ausencia en casi la totalidad de los muestreos realizados en cursos de menor entidad, en su mayoría afluentes del propio río Ebro.

El hábitat que ocupa A. murcius en el alto Ebro coincide con el descrito en otras localidades de la especie o de su congénere A. occidentalis (Nieser et al., 1994; Miguélez \& Valladares, 2006; Carbonell, 2009): tramos de río donde dominan las tablas rápidas y las corrientes, de cauces anchos entre 20-30 m; profundidades entre los 50-90 cm; velocidad media de la corriente en la columna de agua alta, alrededor de $1 \mathrm{~m} / \mathrm{s}$;

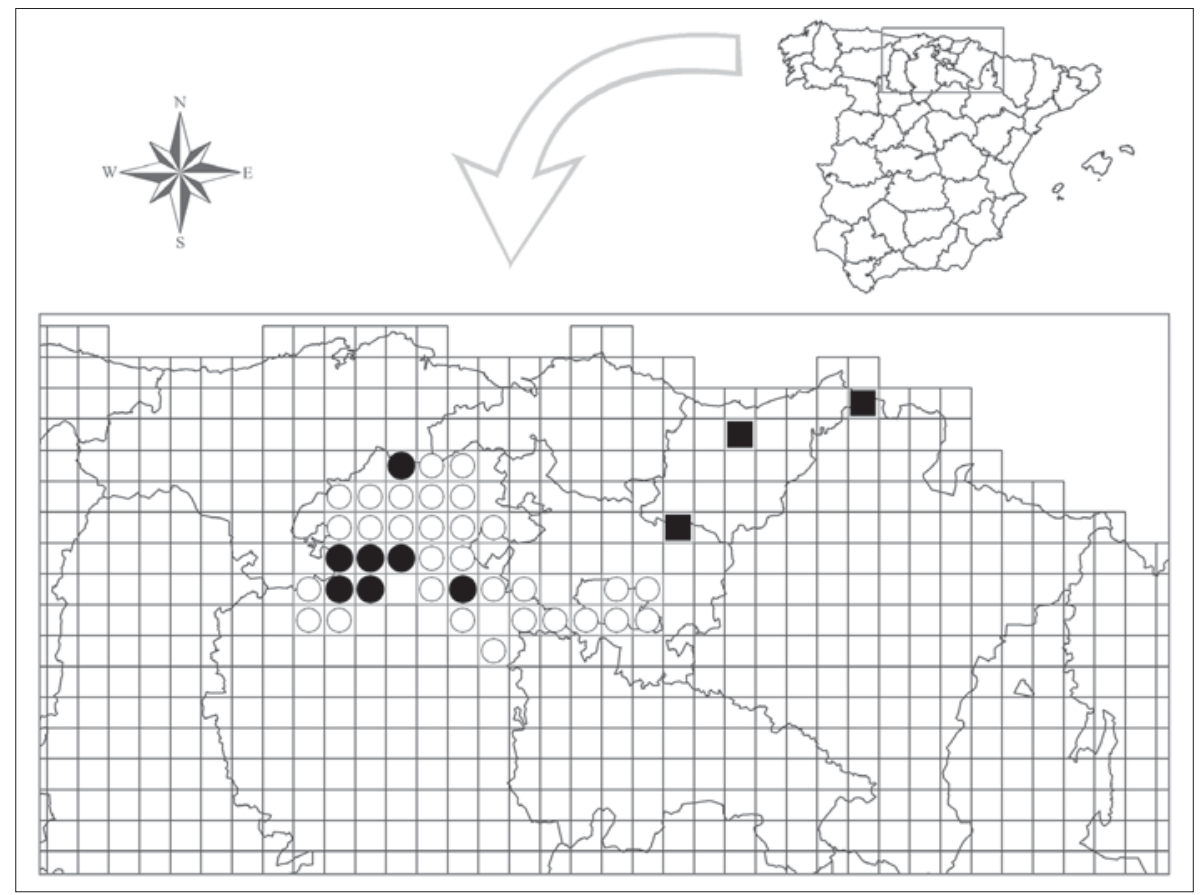

Figura 1. Distribución de Aphelocheirus murcius Nieser \& Millán, 1989 en el norte de la Península Ibérica (cuadrículas UTM de $10 \times 10 \mathrm{~km}$ ). • citas nuevas; o muestreo negativo; citas bibliográficas. Aphelocheirus murcius Nieser \& Millán, 1989 distribution, in the north of the Iberian Peninsula (UTM squares $10 \times 10 \mathrm{~km}) \bullet$ new records; ○ negative sampling; $\mathbf{a}$ bibliographic records. 
el sustrato dominante son cantos y gravas, entre 7-30 cm de diámetro, acompañado de piedras $\mathrm{y}$ arenas. Las localidades en las que se ha encontrado esta especie se corresponden con tramos medios y regulados, principalmente por el embalse del Ebro. El rango altitudinal está entre 520 y 668 m s.n.m., con la excepción de la localidad del río Trueba, tramo sin regulación y relativamente alto (772 m s.n.m.).

Todas las localidades presentan un muy buen estado ecológico de sus aguas. Así, los valores de algunos parámetros biológicos como el índice IBMWP se sitúa entre 163-249 puntos (media 202), el IASPT entre 5.1-6.3 (media 5.7) y las medias de riqueza de taxones y riqueza EPT es superior a 35 y 12 taxones respectivamente. Los parámetros fisicoquímicos no reflejan evidencias de contaminación orgánica u otras perturbaciones, con concentraciones de nitritos, nitratos y amonio muy bajas en relación a los niveles establecidos en la Directiva 78/659/CEE. Los valores medios de $\mathrm{pH}$ y conductividad son 8.2 (rango entre 8.0 y 8.7 ) y $413 \mu \mathrm{S} \mathrm{cm}^{-1}$ (rango $300-602 \mu \mathrm{S} \mathrm{cm}^{-1}$ ) que parecen indicar su preferencia por las aguas básicas (Carbonell, 2009), asociadas a la abundante litología calcárea del alto Ebro. Otro parámetro, la concentración de oxígeno disuelto, se encuentra entre los 8.75 y $11.30 \mathrm{mg} / \mathrm{l}$ (media $9.88 \mathrm{mg} / \mathrm{l}$ ), que como en el resto especies del género en Europa, requiere de aguas bien oxigenadas (Nieser et al., 1994). La cobertura de vegetación sumergida se halla entre el $10-50 \%$ (media $40 \%$ ), en la cual predominan especies de los géneros Ranunculus y Myriophyllum. El grado de conservación de la vegetación de ribera asociada a estos cursos fluviales es excelente, como demuestran los valores del índice de calidad de ribera (QBR) ya que 14 de 18 estaciones superan los 90 puntos y 9 de ellas alcanzan el máximo de 100, con una buena cobertura forestal en las orillas, aunque el porcentaje de sombra sobre la lámina de agua es bajo debido a la anchura del cauce (rango 3-38\%, media $15 \%)$. La vegetación de ribera está compuesta principalmente por aliso (Alnus glutinosa), sauces (Salix spp.) y chopo (Populus sp.), acompañados por otras especies de porte arbóreo y una rica comunidad de especies arbustivas. La especie se encuentra, por tanto, en tramos medios y bien conservados de cursos fluviales con sustrato calcáreo.

A nivel nacional, A. murcius, presenta un alto grado de vulnerabilidad por el carácter restringido y fragmentado de su área de distribución. Se trata de una rareza geográfica, y los ecosistemas que ocupa sufren una importante pérdida en la calidad de su hábitat (Carbonell, 2009), siendo los Aphelocheiridae excepcionales indicadores del estado ecológico de las aguas fluviales (Alba-Tercedor et al., 2002). No obstante, el elevado número de ejemplares capturados en Burgos, un total de 1660 , indica a priori, un buen estado de conservación de las poblaciones, al menos en este tramo del río Ebro. La captura de 256 adultos (sex ratio de 1:1.3) y 1404 ninfas en diferentes estados de desarrollo, revela una aparente buena estructura de la población por sexos y clases de edad. Si bien el número de ejemplares capturados a partir de la localidad de Valdenoceda (antepenúltima estación con capturas) es cada vez más escaso, es posible que las fuertes detracciones de caudal y los dudosos caudales ecológicos (con fuerte estiaje en algunos tramos) a los que se ve sometido el río Ebro, afecten muy negativamente a sus poblaciones. Actualmente estos aprovechamientos hidroeléctricos y sus impactos en el medio fluvial parecen ser la principal amenaza en la zona para las poblaciones de esta especie.

\section{AGRADECIMIENTOS}

Nuestro agradecimiento a David Pérez, Eva Álvarez, Gustavo González, Pilar Canal, Isabel Roa y Rubén González por su ayuda durante el trabajo de campo. A Andrés Millán y José Antonio Carbonell por la revisión de varios ejemplares y la bibliografía facilitada sobre esta especie, y a Javier García y Susana Blanco por la ayuda prestada durante la elaboración del trabajo.

\section{BIBLIOGRAFÍA}

ALBA-TERCEDOR J., P. JÁIMEZ-CUÉLLAR, M. ÁlVAREZ, J. AVILÉS, N. BONADA, J. CASAS, A. MELLADO, M. ORTEGA, I. PARDO, 
N. PRAT, M. RIERADEVALL, S. ROBLES, C. E. SÁINZ-CANTERO, A. SÁNCHEZ-ORTEGA, M. L. SUÁREZ, M. TORO, M. R. VIDAL-ABARCA, S. VIVAS \& C. ZAMORA-MUÑOZ. 2002. Caracterización del estado ecológico de ríos mediterráneos ibéricos mediante el índice IBMWP (antes BMWP'). Limnetica, 21(3-4): 175-185.

AUKEMA, B. \& C. RIEGER. 1995. Catalogue of the Heteroptera of the Paleartic Region. Vol. 1: Enicocephalomorpha, Dipsocoromorpha, Nepomorpha, Gerromorpha, and Leptopodomorpha. Netherlands Entomological Society, Amsterdam, 222 pp.

BAENA, M. \& M. A. VÁZQUEZ. 1986. Catálogo preliminar de los Heterópteros acuáticos ibéricos (Heteroptera: Nepomorpha, Gerromorpha). Graellsia, 42: 61-89.

CARBONELL, J. A. 2009. El género Aphelocheirus Westwood, 1833 (Hemiptera: Aphelocheiridae) en la Península Ibérica: estado de conservación y valor indicador. Trabajo Fin de Master, Universidad de Murcia, Murcia, 38 pp.

FERNÁNDEZ BERNALDO DE QUIRÓS, C. 1982. Heterópteros acuáticos y semiacuáticos de Asturias (N. España). I. Catálogo sistemático. Bol. $R$. Soc. Esp. Hist. Nat. (Biol.), 80: 211-218.

JÁIMEZ-CUÉLLAR P., S. VIVAS, N. BONADA, S. ROBLES, A. MELLADO, M. ÁllVAREZ, J. AVILÉS, J. CASAS, M. ORTEGA, I. PARDO, N. PRAT, M. RIERADEVALL, C. E. SÁINZCANTERO, A. SÁNCHEZ-ORTEGA, M. L. SUÁREZ, M. TORO, M. R. VIDAL-ABARCA, C. ZAMORA-MUÑOZ \& J. ALBA-TERCEDOR. 2002. Protocolo GUADALMED (PRECE) Limnetica, 21(3-4): 187-204.

JÁIMEZ-CUÉLLAR P., P. PALOMINO-MORALES, J. LUZÓN-ORTEGA \& J. ALBA-TERCEDOR. 2006. Comparación de metodologías empleadas para la evaluación del estado ecológico de los cursos de agua. Implicaciones sobre la obtención de datos de composición y abundancia según la Directi- va Marco del Agua. Tecnología del Agua, 26: 42-57. MIGUÉLEZ, D. \& L. F. VALLADARES. 2006. Nuevos datos sobre la distribución geográfica y el hábitat de Aphelocheirus occidentalis Nieser \& Millán, 1989 (Hemiptera: Aphelocheiridae). Boln. SEA, 38: 343-344.

MILLÁN, A., J. L. MORENO \& J. VELASCO. 2002. Los Coleópteros y Heterópteros acuáticos y semiacuáticos de la provincia de Albacete. Catálogo faunístico y estudio ecológico. Instituto de Estudios Albacetenses "Don Juan Manuel", Excma. Diputación Provincial de Albacete, Albacete, 180 pp.

MURILLO, J. 1985. Algunes captures d'Heteroptèrs aquàtics efectuades a Catalunya i altres localitats de la resta de la Península Ibérica. Bull. Inst. Cat. Hist. Nat., 52 (Sec. Zool., 6: 139-147.

NIESER, N., M. BAENA, J. MARTÍNEZ-AVILÉS \& A. MILLÁN. 1994. Claves para la identificación de los Heterópteros acuáticos (Nepomorpha \& Gerromorpha) de la Península Ibérica -Con notas sobre las especies de las Islas Azores, Baleares, Canarias y Madeira. Claves de identificación de la Flora y Fauna de las aguas continentales de la Península Ibérica $n^{0}$ 5. Asociación Española de Limnología, Madrid, 112 pp.

NIESER, N. \& A. MILLÁN. 1989. Two new species of Aphelocheirus from the Iberian Peninsula (Heteroptera: Naucoridae). Ent. Ber. Amst., 49(8): 11-117.

NIESER, N. \& C. MONTES. 1984. Lista faunística y bibliográfica de los Heterópteros acuáticos (Nepomorpha \& Gerromorpha) de España y Portugal. Listas de la Flora y Fauna de las aguas continentales de la Península Ibérica ${ }^{0} 1$. Asociación Española de Limnología, Madrid, 69 pp.

SEABRA, A. F. DE 1926. Hémiptères Hétéroptères de la province de Tras-os-Montes. Mem. Est. Mus. Zool. Univ. Coimbra, Ser. 1, 8: 5-39.

SEABRA, A. F. DE 1939. Contribuição para a história da entomologia em Portugal. Publicaçoes $D i$ rec. Ger. Serv. Flor. Aquic., 6(2): 155-302. 